\title{
Nonlinear Interaction of Elliptical q-Gaussian Laser Beams with Plasmas with Axial Density Ramp: Effect of Ponderomotive Force
}

Naveen Gupta ( $\square$ naveen.21559@lpu.co.in )

Lovely Professional University

Sandeep Kumar

Lovely Professional University

\section{Research Article}

Keywords: q-Gaussian laser beams, variational theory

Posted Date: March 29th, 2021

DOI: https://doi.org/10.21203/rs.3.rs-307559/v1

License: (c) (1) This work is licensed under a Creative Commons Attribution 4.0 International License.

Read Full License 


\title{
Nonlinear Interaction of Elliptical q-Gaussian Laser Beams with Plasmas with Axial Density Ramp: Effect of Ponderomotive Force
}

\author{
Naveen Gupta, Sandeep Kumar \\ Lovely Professional University, Phagwara \\ Email :- naveens222@rediffmail.com, sandy.dadra@yahoo.com
}

\begin{abstract}
Theoretical investigation on optical self action effects of intense $q$-Gaussian laser beams interacting with collisionless plasmas with axial density ramp has been presented. Emphasis are put on investigating the dynamics of beam width and axial phase of the laser beam. Effect of the ellipticity of the cross section of the laser beam also has been incorporated. Using variational theory based on Lagrangian formulation nonlinear partial differential equation (P.D.E) governing the evolution of beam amplitude has been reduced to a set of coupled ordinary differential equations for the beam widths of the laser beam along the transverse directions. The evolution equation for the axial phase of the laser beam has been obtained by the Fourier transform of the amplitude structure of the laser beam from coordinate space to $\left(k_{x}, k_{y}\right)$ space. The differential equations so obtained have been solved numerically to envision the effect of laser-plasma parameters on the propagation dynamics of the laser beam.
\end{abstract}

\section{Introduction}

Laser[1] is the one of the most important scientific inventions of the 20th century. When laser made its debut, it was referred to as solution in search of a problem. Today laser has become ubiquitous in consumer technology, from CD players to supermarket check out scanners. Higher end applications of lasers are also abound. This includes medical diagnostics and treatment[2], nuclear fusion[3], particle accelerators[4], decommissioning of explosives[5] etc. The diversity in the applications of lasers can be felt from the fact that currently this instrument is being used for heating as well as for ultra intense cooling. The same instrument can produce extremely hot state of matter[6] (plasma) as well 
as extremely cold state of matter[7] (Bose Einstein Condensate). The impact of laser on society has changed over time, and is still changing. Already, lasers have provided the preferred solution to an impressive variety of real world situations, and it is expected that in coming years it will keep on enhancing quality of life and will contribute wealth to the world economy.

In most of the applications the laser intensity is the key parameter that decides their ultimate breath. Currently, due to the light's inherent wave property to diffract, the laser power has gotten into bottleneck at the order of few peta watts. Initially it was believed that diffraction of the laser beam can not be avoided during its propagation neither through vacuum nor through material media. However, in 1964 Chio et al[8] showed that in media whose index of refraction depends on the intensity of light, the spreading of an optical beam in principle can be obviated. Hence, the expansion of optical beam due to diffraction is neither inevitable nor irreducible.

Self-focusing and self-trapping are two examples of nonlinear optical effects which may arise from one of many physical mechanisms. Self-focusing describes the formation of a light induced channel in an illuminated material which confines the optical beam. This channel serves as a lens. Selftrapping occurs when self-focusing substantially exactly counteracts beam spreading due to diffraction. When this happens, the cross section of the light induced channel remains substantially constant with propagation distance over the distance of the self-trapping. Other similar mechanisms also exist. For example, a modified self-trapping effect occurs when self-focusing is somewhat larger than beam spreading due to diffraction. In that case the cross section of the beam vary in an oscillatory way i.e., it remains constant on average. In general, the diameter of a trapped beam may be slightly modulated along the propagation direction, as if wave guiding by the medium were due to a periodic sequence of convex lenses. This results in a channel with diameter variations. In this case, self-focusing does not exactly balance diffraction point-by-point along the longitudinal direction. Nevertheless, on average, the beam is trapped.

A material exhibits self-trapping or self-focusing when the index of refraction changes in the pres- 
ence of optical radiation in a way to induce waveguiding of that same optical beam which causes the index of refraction to change. Plasmas can produce self focusing of laser beams mainly through three mechanisms. These mechanisms are (1) Relativistic self focusing[13,14] (2) Ponderomotive Self focusing[15-17] (3) nonlinear Ohmic heating[18,19]. In relativistic mechanism modification in optical properties of plasma occurs without any modification in electron density whereas in the remaining two mechanisms this modification occurs via redistribution of plasma electrons. The relativistic nonlinearity immediately comes into picture when the power of incident laser exceeds the threshold required for self-focusing and thus it does not show any transient behaviour. In this mechanism optical properties of plasma become a function of intensity of the electromagnetic beam due to change in electron mass when it starts oscillating at a velocity comparable to that of light. In ponderomotive force mechanism an electron density gradient is produced in the illuminated portion of the plasma due to the migration of electrons from high to low intensity regions. Thus, the refractive index of the region of plasma illuminated by the laser beam resembles to that of graded index fiber. Ohmic heating of electrons can also modifies the electron density and thus refractive index of plasma. In this mechanism also, the modification of index of refraction occurs due to redistribution of electrons. But this time electrons migrate from the high intensity regions due to the temperature gradient produced by the laser beam in the illuminated portion of plasma.

Self focusing of the laser beams in different nonlinear media has been a hot topic of research since its discovery by Askaryan[11]. In past few years a vast literature has been reported by researchers from all over the globe on various aspects of this phenomenon[20-23]. Early seminal work of Sodha et al[24] gave a gravest blow to the investigation of this phenomenon for intense laser beams interacting with plasmas in different environments and regimes. Specially in context of inertial confinement fusion this phenomenon is at the vanguard of theoretical as well as experimental investigations[25].

Laser beams differing in irradiance over their cross sections behave differently in plasmas[26]. However, from literature review it has been seen that in previous investigations on self action effects of 
elliptical laser beams in plasmas it has been assumed that the laser system operating in lowest order mode i.e., TEM $_{00}$ mode the out put beam is ideally Gaussian[27-30]. However, the experimental findings of Patel et al[31] reveal that even for TEM 00 mode the out put beam is not exactly Gaussian. A large portion of the laser energy was fount to be present outside the full width half maximum of the intensity profile. By fitting into experimental data[32] it was suggested that the actual profile of the laser beam can be modeled by a class of distribution functions known as Tsalli's $q$-Gaussian distribution[33]. Since, no experimental or theoretical investigation on self focusing of elliptical $q$-Gaussian laser beams in collisionless plasmas has been reported till date, this gave us a strong motivation to investigate the same. Thus this paper aims to present theoretical investigation on self action effects of elliptical q-Gaussian laser beam in collisionless plasmas with axial density ramp.

\section{Characteristics of $q$-Gaussian Laser Beam}

The amplitude structure over the cross section of elliptical $q$-Gaussian laser beam at the plane if incidence (i.e., $z=0$ ) is given by $[34,35]$

$$
A_{0}(x, y)=E_{00}\left\{1+\frac{1}{q}\left(\frac{x^{2}}{a^{2}}+\frac{y^{2}}{b^{2}}\right)\right\}^{-\frac{q}{2}}
$$

where, $E_{00}$ is the axial amplitude of the field of the laser beam and $a, b$ are the widths of the laser beam in $x, y$ directions, respectively. The phenomenological parameter $q$ is related to the deviation of amplitude structure from ideal Gaussian profile and is termed as deviation parameter. The value of deviation parameter $q$ varies from laser to laser and can be obtained by fitting into the experimental data for a given laser system.

The amplitude structure of the laser beam deviates from the ideal Gaussian distribution due to cavity imperfections like misalignment of the end mirrors, non uniformity of the reflectivity of the mirrors or inclusion of impurities into the gain medium. Due to these imperfections a significant amount of laser energy exists in the off axial part of the beam. E.g., in case of Vulcan petawatt laser 
it has been observed that $20 \%$ energy is contained within $6.9 \mu \mathrm{m}$ FWHM and $50 \%$ energy is within $16 \mu m$ FWHM.

As the value of $q$ increases the amplitude structure of the laser beam converges towards the ideal Gaussian and becomes exactly Gaussian for $q=\infty$ i.e.,

$$
\lim _{q \rightarrow \infty} A_{0}(x, y)=E_{00} e^{-\left(\frac{x^{2}}{2 a^{2}}+\frac{y^{2}}{2 b^{2}}\right)}
$$

In order to see the effect of deviation parameter $q$ on amplitude structure of the laser beam, we have shown the variation of normalized amplitude $\frac{A_{0}(x, y)}{E_{00}}$ with $\frac{x}{a}$ in fig.(1), while keeping $y=0$. It can be seen that $q$-Gaussian distribution is characterized by expanded wings as compared to the Gaussian distribution. These expanded wings of the distribution correspond to the beam energy that lies ion the off axial part of the beam as can be seen from fig.(2). Fig.(2) portray the actual irradiance over the cross section of the laser beams for different values of deviation parameter $q$. Again it can be seen that the deviation parameter $q$ does not affect the irradiance in the region near to the beam axis however, it significantly affects the off axial parts of the beam. With increase in the deviation parameter $q$ the off axial part of the beam becomes weaker.

For $z>0$, the energy conserving ansatz for the amplitude structure of the laser beam is given by

$$
A_{0}(x, y, z)=\frac{E_{00}}{\sqrt{f_{x} f_{y}}}\left\{1+\frac{1}{q}\left(\frac{x^{2}}{a^{2} f_{x}^{2}}+\frac{y^{2}}{b^{2} f_{y}^{2}}\right)\right\}^{-\frac{q}{2}}
$$

where, $f_{x}$ and $f_{y}$ are the currently undetermined, real functions of only the longitudinal coordinate $z$. Upon multiplication with $a$ and $b$, respectively, these functions give the instantaneous widths of the beam in $x$ and $y$ directions, respectively. Thus, $f_{x}$ and $f_{y}$ are termed as dimensionless beam width parameters.

Further in order to see the effect of deviation parameter $q$ on effective width of the beam, we make use of spatial intensity moments of the laser beam. In this regard the effective cross sectional width of the beam is defined in root mean square sense as[36]

$$
<a^{2}>=<x^{2}>+<y^{2}>
$$


where,

$$
\begin{aligned}
& <x^{2}>=\frac{1}{I_{0}} \iint A_{0} x^{2} A_{0} d^{2} r \\
& <y^{2}>=\frac{1}{I_{0}} \iint A_{0} y^{2} A_{0} d^{2} r
\end{aligned}
$$

with

$$
I_{0}=\iint A_{0} A_{0} d^{2} r
$$

and

$$
d^{2} r=d x d y
$$

Using above definition of effective beam width, we get the r.m.s beam width of elliptical $q$-Gaussian beam as

$$
<a^{2}>=\frac{1}{2}\left(1-\frac{2}{q}\right)^{-q}\left(a^{2} f_{x}^{2}+b^{2} f_{y}^{2}\right)
$$

The effective beam width of corresponding Gaussian beam $\left(A_{G}(x, y, z)=\frac{E_{00}}{\sqrt{f_{x} f_{f}}} e^{-\left(\frac{x^{2}}{2 a^{2} f_{x}^{2}}+\frac{y^{2}}{2 b^{2} f_{y}^{2}}\right)}\right)$ is

$$
<a^{2}>_{G}=\frac{1}{2}\left(a^{2} f_{x}^{2}+b^{2} f_{y}^{2}\right)
$$

Thus, the ratio $\Sigma$ of r.m.s beam width of $q$-Gaussian beam to that of Gaussian beam is

$$
\Sigma=\left(1-\frac{2}{q}\right)^{-q}
$$

Fig.(3) depicts the variation of effective beam with of $q$ gaussian laser beam with deviation parameter q. It can be seen that deviation parameter $q$ significantly affects the effective cross section of the laser beam. With increase in the value of $q$ the effective cross section of the laser beam gets shrinked. This is due to the fact that off axial parts of the laser beam with higher value of $q$ are weaker compared to that with beams with lower value of $q$. 


\section{Ponderomotive Nonlinearity of Plasma}

Consider the propagation of a laser beam with electric filed vector

$$
\mathbf{E}(\mathbf{r}, \mathbf{t})=A_{0}(x, y, z) e^{-\iota\left(k_{0} z-\omega_{0} t\right)} \mathbf{e}_{\mathbf{x}}
$$

through a collisionless plasma whose equilibrium electron density increases along longitudinal direction as $n_{0}(z)=n_{0}(1+\tan (d z))$, where, $\left(k_{0}, \omega_{0}\right)$ are the vacuum wave number and angular frequency of the laser beam, respectively, $n_{0}$ is the electron density at at $z=0$ and the constant $d$ is associated with the rate of increase of electron density with distance and hence is termed as slope of the density ramp. Due to the amplitude structure over the cross section of the laser beam given by eq.(2) the plasma electrons experience a ponderomotive force given by[16,24]

$$
F_{P}=-\frac{e^{2}}{4 m \omega_{0}^{2}} \nabla\left(A_{0} A_{0}^{\star}\right)
$$

Here, $(e, m)$ are the electronic charge and mass, respectively. As this ponderomotive force is proportional to the negative of the intensity gradient of the laser beam, this force causes the evacuation of electrons from high intensity regions of the illuminated portion of plasma. The modified electron density of the plasma is given by[24]

$$
n=n_{0}(z) e^{-\frac{e^{2}}{8 m \omega_{0}^{2} T_{0} K_{0}} A_{0} A_{0}^{\star}}
$$

where, $T_{0}$ is the temperature of plasma and $K_{0}$ is the Boltzmann constant. This modified electron density in turn alters the dielectric function $\left(\epsilon=1-\frac{4 \pi e^{2} n}{m \omega_{0}^{2}}\right)$ of plasma as

$$
\epsilon=1-\frac{\omega_{p 0}^{2}}{\omega_{0}^{2}}(1+\tan (d z)) e^{-\frac{e^{2}}{8 m \omega_{0}^{2} T_{0} K_{0}} A_{0} A_{0}^{\star}}
$$

where, $\omega_{p 0}^{2}=\frac{4 \pi e^{2}}{m} n_{0}$ is the unperturbed plasma frequency i.e., the plasma frequency in the absence of laser beam.

Thus, the ponderomotive force on the plasma electrons produced by the laser beam, makes the index of refraction of plasma intensity dependent which in turn due to the spatial dependence of 
the amplitude structure of the laser beam, resembles to that of graded index fiber. Separating the dielectric function of plasma into linear $\left(\epsilon_{0}\right)$ and nonlinear $(\phi)$ parts as

$$
\epsilon=\epsilon_{0}+\phi\left(A_{0} A_{0}^{\star}\right)
$$

we get

$$
\epsilon_{0}=1-\frac{\omega_{p 0}^{2}}{\omega_{0}^{2}}
$$

and

$$
\phi\left(A_{0} A_{0}^{\star}\right)=\frac{\omega_{p 0}^{2}}{\omega_{0}^{2}}(1+\tan (d z))\left\{1-e^{-\frac{e^{2}}{8 m \omega_{0}^{2} T_{0} K_{0}} A_{0} A_{0}^{\star}}\right\}
$$

\section{Evolution of Beam Width of Laser Beam}

The propagation of an optical beam through a nonlinear medium characterized by nonlinear dielectric function $\phi\left(A_{0} A_{0}^{\star}\right)$ is governed by wave equation[16]

$$
2 \iota k_{0} \frac{\partial A_{0}}{\partial z}=\nabla_{\perp}^{2} A_{0}+\frac{\omega_{0}^{2}}{c^{2}} \phi\left(A_{0} A_{0}^{\star}\right) A_{0}
$$

Eq.(10) is very much identical Schrodinger equation encountered in quantum mechanics. The only difference being that here time coordinate 't' has been replaced with space coordinate ' $z$ ' and here the potential function $\phi$ itself is dependent on the field amplitude $A_{0}$. Hence eq.(10) is also known as nonlinear Schrodinger wave equation (NLSE).

Being nonlinear in nature, superposition principle does not apply to eq.(10) i.e., linear combination of two solutions is not a solution. Mathematically this means that conventional method of solving partial differential equations i.e., expansion in power series are not applicable to NSWE. In fact no exact analytical solution exists for this equation. In order to obtain physical insight into the propagation dynamics of the laser beam we use a semi analytical technique known as variational method $[37,38]$. This method converts the problem of solving a partial differential equation to that of 
solving a set of coupled ordinary differential equations. These ordinary differential equations govern the evolution of the various parameters of interest. In case of self focusing of laser beams the parameter of interest is the beam width of the laser beam. According to this method eq.(10) is a variational problem for action principle based on Lagrangian density

$$
\mathcal{L}=\iota\left(A_{0} \frac{\partial A_{0}^{\star}}{\partial z}-A_{0}^{\star} \frac{\partial A_{0}}{\partial z}\right)+\left|\nabla_{\perp} A_{0}\right|^{2}-\frac{\omega_{0}^{2}}{c^{2}} \int^{A_{0} A_{0}^{\star}} \phi\left(A_{0} A_{0}^{\star}\right) d\left(A_{0} A_{0}^{\star}\right)
$$

Substituting the trial function given by eq.(2) in lagrangian density and integrating over the entire cross section of the laser beam we get the reduced lagrangian as $L=\int \mathcal{L} d^{2} r$. The corresponding Euler-Lagrange equations

$$
\frac{d}{d z}\left(\frac{\partial L}{\partial\left(\frac{\partial f_{x, y}}{\partial z}\right)}\right)-\frac{\partial L}{\partial f_{x, y}}=0
$$

give

$$
\begin{aligned}
& \frac{d^{2} f_{x}}{d z^{2}}=\frac{1}{2 k_{0}^{2} a^{4}} \frac{1}{f_{x}^{3}}\left(1-\frac{1}{q}\right)\left(1-\frac{2}{q}\right)\left[\left(1+\frac{1}{q}\right)^{-1}+\left(\frac{<L_{1}>}{E_{00}^{2}} f_{x} f_{y}+\frac{2 E_{00}^{2}}{f_{x}^{2} f_{y}} \frac{\partial<L_{1}>}{\partial f_{x}}\right)\right] \\
& \frac{d^{2} f_{y}}{d z^{2}}=\frac{1}{2 k_{0}^{2} b^{4}} \frac{1}{f_{y}^{3}}\left(1-\frac{1}{q}\right)\left(1-\frac{2}{q}\right)\left[\left(1+\frac{1}{q}\right)^{-1}+\left(\frac{<L_{1}>}{E_{00}^{2}} f_{x} f_{y}+\frac{2 E_{00}^{2}}{f_{x} f_{y}^{2}} \frac{\partial<L_{1}>}{\partial f_{y}}\right)\right]
\end{aligned}
$$

where,

$$
<L_{1}>=\frac{\omega_{0}^{2}}{c^{2}} \int\left(\int^{A_{0} A_{0}^{\star}} \phi\left(A_{0} A_{0}^{\star}\right) d\left(A_{0} A_{0}^{\star}\right)\right) d^{2} r
$$

Eqs.(13) and (14) can be written as

$$
\begin{aligned}
& \frac{d^{2} f_{x}}{d z^{2}}=\frac{1}{2 k_{0}^{2} a^{4} f_{x}^{3}} \frac{\left(1-\frac{1}{q}\right)\left(1-\frac{2}{q}\right)}{\left(1+\frac{1}{q}\right)}+\frac{1}{2} \frac{\left(1-\frac{2}{q}\right)}{a^{2} \epsilon_{0} I_{0}} \int x A_{0} A_{0}^{\star} \frac{\partial \phi}{\partial x} d^{2} r \\
& \frac{d^{2} f_{y}}{d z^{2}}=\frac{1}{2 k_{0}^{2} b^{4} f_{y}^{3}} \frac{\left(1-\frac{1}{q}\right)\left(1-\frac{2}{q}\right)}{\left(1+\frac{1}{q}\right)}+\frac{1}{2} \frac{\left(1-\frac{2}{q}\right)}{b^{2} \epsilon_{0} I_{0}} \int y A_{0} A_{0}^{\star} \frac{\partial \phi}{\partial y} d^{2} r
\end{aligned}
$$

Using eqs.(2) and (9) in eqs.(15) and (16) we get

$$
\frac{d^{2} f_{x}}{d \xi^{2}}=\frac{\left(1-\frac{1}{q}\right)\left(1-\frac{2}{q}\right)}{\left(1+\frac{1}{q}\right)} \frac{1}{f_{x}^{3}}-\left(1-\frac{1}{q}\right)\left(1-\frac{2}{q}\right)\left(\frac{\omega_{p 0}^{2} a^{2}}{c^{2}}\right)\left(1+\tan \left(d^{\prime} \xi\right)\right) \frac{\beta E_{00}^{2}}{f_{x}^{2} f_{y}} I
$$




$$
\frac{d^{2} f_{y}}{d \xi^{2}}=\left(\frac{a}{b}\right)^{4} \frac{\left(1-\frac{1}{q}\right)\left(1-\frac{2}{q}\right)}{\left(1+\frac{1}{q}\right)} \frac{1}{f_{y}^{3}}-\left(\frac{a}{b}\right)^{2}\left(1-\frac{1}{q}\right)\left(1-\frac{2}{q}\right)\left(\frac{\omega_{p 0}^{2} a^{2}}{c^{2}}\right)\left(1+\tan \left(d^{\prime} \xi\right)\right) \frac{\beta E_{00}^{2}}{f_{x} f_{y}^{2}} I
$$

where,

$$
\begin{gathered}
\beta=\frac{e^{2}}{8 m \omega_{0}^{2} T_{0} K_{0}} \\
d^{\prime}=d k_{0} a^{2} \\
\xi=\frac{z}{k_{0} a^{2}} \\
I=\int_{0}^{\infty} t\left(1+\frac{t}{q}\right)^{-2 q-1} e^{-\frac{\beta E_{00}^{2}}{f_{x} f_{y}}\left(1+\frac{t}{q}\right)^{-q}} d t
\end{gathered}
$$

Eqs.(17) and (18) are the nonlinearly coupled differential equations governing the evolution of beam widths of the elliptical $q$-Gaussian laser beams along $x$ and $y$ directions with longitudinal distance of propagation. It can be seen that the although in linearly media the beam widths of the laser beam along the two transverse directions evolve independently, however due the laser induced ponderomotive force they get coupled to each other. The set of eqs.(17) and (18) also indicate that variational theory has reduced the original problem of solving a nonlinear partial differential equation to a set of coupled ordinary differential equations. Also, this reduced set of coupled differential equations is also lacking from an exact closed form solution, its approximate solution can be easily obtained by simple numerical techniques. In the present investigation these equations have been solved with the help of Runge Kutta fourth order method for following set of laser-plasma parameters:

$$
\omega_{0}=1.78 \times 10^{15} \mathrm{rad} / \mathrm{sec}, a=10 \mu \mathrm{m}, \beta E_{00}^{2}=3, \frac{\omega_{p 0}^{2} a^{2}}{c^{2}}=9
$$

and for different values of $q, d^{\prime}$ and $\frac{a}{b}$ viz.,

$$
q=(3,4, \infty), d^{\prime}=(0.25,0.35,0.45) \text { and } \frac{a}{b}=(1,1.1,1.2)
$$

under the boundary condition that at the plane of incidence the laser beam is having plane wavefront. Mathematically this condition means that at $\xi=0$ :

$$
f_{x, y}=1
$$




$$
\frac{d f_{x, y}}{d \xi}=0
$$

Firstly, in order to see the linear propagation of $q$-Gaussian laser beam, eqs.(17) and (18) have been solved in the absence of plasma medium for different values of deviation parameter $q$ and the corresponding variations of the beam width have been sown in figs.(4) and (5). It can be seen that irrespective of the ellipticity of the beam or deviation parameter $q$, the beam widths of the laser beam along both the transverse directions broaden with distance of propagation. This is due to the fact that a laser beam with finite cross section can be considered as a superposition of plane waves, all having the same wave number, but with different angle with respect to the beam axis. Therefore, each component propagates at different phase velocity with respect to the longitudinal direction. Thus, each plane wave acquires a different phase and thus the beam broadens along the transverse directions.

It can also be seen (fig.4) that although in vacuum the beam widths along $x$ and $y$ directions broaden independently of each other, the beam width along $y$ direction broaden at a faster rate compared to that along $x$ direction. This is due to the fact that diffraction effect of the laser beam varies inversely as the square of the transverse spread of the laser beam and in fig.(4) we have taken $\frac{a}{b}=1.1$ which means $a>b$. As, a result the diffraction effect is more prominent along $y$ direction compared to that along $x$ direction.

The plots in fig.(5) depict that with increase in the value of deviation parameter $q$ the diffraction broadening of the laser beam along both the transverse direction increases. This is due to the fact that as the value deviation parameter increases the over all cross section of the laser beam gets shrinked. Decrease in the effective spot size of the laser beam results in its enhanced diffraction with increase in deviation parameter $q$. Thus, it can be concluded that although the amplitude structure of the laser beam deviates from ideal Gaussian profile due to cavity imperfections, but it helps to obviate the diffraction broadening of the beam i.e., $q$-Gaussian beams are more directional compared to ideal Gaussian beams.

Now in order to see the effect of deviation parameter $q$ on the evolution of beam widths of the laser 
beam in plasma medium, we have solved eqs.(17) and (18) for different values of $q$ in the presence of plasma medium and the corresponding evolutions of the beam widths along $x$ and $y$ directions with longitudinal distance are sown in figs.(6) and (7). It can be seen that inside the plasma medium the beam widths of the laser beam along both the transverse directions show oscillatory behaviour over the longitudinal direction. This behaviour of the laser beam can be explained by analyzing the role and origin of various terms contained in the evolution equations for the beam widths i.e., eqs.(17) and (18). The first terms on the right hand side (R.H.S) of these equations that vary inversely as the cube of corresponding beam width (i.e., as $f_{x, y}^{-3}$ ) are the spatial dispersive term that model the spreading of the laser beam in transverse $x$ and $y$ directions as a consequence of the diffraction divergence. The second terms on the R.H.S of these equations that have complex dependence on beam widths $f_{x, y}$ originate as a consequence of ponderomotive force exerted by the laser beam on plasma electrons. These terms model the nonlinear refraction of the laser beam and the nonlinear coupling of the beam widths along transverse directions. As a result of laser induced nonlinearity of plasma the resulting nonlinear refraction of the laser beam tends to counter balance the effect of diffraction along both the transverse directions. Thus, during the propagation of laser beam through the plasma, there starts a competition between the two phenomena of diffraction and nonlinear refraction. The winning phenomenon decides the ultimate behaviour of the laser beam i.e., the whether the beam will converge or diverge. Thus, there exists a critical value of beam intensity (that can be obtained by balancing the two terms on the R.H.S of eqs. 17 and 18) above which the beam converges along both the transverse directions. In the present investigation the initial beam intensity has been taken to be greater than the critical intensity. That is why the beam widths of the laser beam along both the transverse directions converging initially. As the cross section of the laser beam shrinks, its intensity increases. When the intensity of the laser beam becomes too high, the illuminated portion of the plasma gets almost evacuated from the electrons. Hence, the beam now propagates as if it is propagating through vacuum. As an optical beam propagating through vacuum undergo diffraction, the beam width of a laser beam propagating 
through plasma, after attaining possible minimum value bounces back towards its original value. As the widths of the laser beam along both the transverse directions start expanding, the competition between diffraction broadening and nonlinear refraction starts again. Now this competition lasts till $f_{x, y}$ obtain their maximum possible values. These, processes keep on repeating themselves and thus give oscillatory behaviour to the beam widths of the laser beam along the two transverse directions.

Further it has been observed that after every focal spot the maximum as well as the minimum of the beam width shift downwards. This is owing to the fact that the equilibrium electron density is an increasing function of longitudinal distance. Hence, the plasma index of refraction keep on decreasing with the penetration of laser beam into the plasma. Consequently, the self focusing effect gets enhanced and the maximum as well as minimum of the beam width go on shifting downwards after every focal spot. It is also seen that the frequency of oscillations of beam with increases with distance. The physics behind this fact is that denser is the plasma, higher will be the phase velocity of laser beam through it. Hence, in denser plasma laser beam takes less duration to get self focused.

It can be also be seen that initially the beam widths of the laser beam along the two transverse directions vary in phase with each other but over some distance of propagation their oscillations establish a phase mismatch. This phase mismatch in the oscillations of beam widths along $x$ and $y$ directions is due to the fact that due its ellipticity the laser beam experience different indices of refraction along $x$ and $y$ directions i.e., for the elliptical beam the plasma behaves as an anisotropic medium.

It can also be seen that the extent of self focusing of the laser beam along $x$ direction is more compared to that along $y$ direction. This is due to the fact that initial width of the beam along $x$ direction is more compared to that along $y$ direction $\left(\frac{a}{b}=1.1\right)$. Thus, the opposition offered by diffraction effect to the nonlinear refraction is more along $y$ direction. This results in reduced focusing along $y$ direction.

The plots in fig.7 depict that with increase in the value of deviation parameter $q$, the extent of 
self focusing along both the transverse directions gets reduced. This is due to the fact for laser beams with larger value of $q$, most of the beam energy is concentrated around a narrow region around the beam axis. Hence, these beams get a little contribution form the off axial rays towards the nonlinear refraction. As the phenomenon of self focusing is a homeostasis of nonlinear refraction of the optical beam due to optical nonlinearity of the medium, increase in the value of deviation parameter $q$ reduces the extent of self focusing of the laser beam. Thus, compared to $q$-Gaussian laser beams, ideal Gaussian laser beams possess minimum focusing character.

It can also be seen that instead of their reduced focusing, laser beams with higher values of deviation parameter $q$ possess faster focusing along both the transverse directions. This is due to the faster focusing character of the rays closer to beam axis. Being away from the beam axis, off axial rays take more duration to get self focused. As there are more number of off axial rays in laser beams with lower values of deviation parameter $q$, these beams possess slower focusing character.

Fig.8 illustrates the effect of beam ellipticity along $y$ direction on self focusing of the laser beam. It can be seen that with increase in the beam ellipticity along $y$ direction there is reduction in extent of self focusing of the laser beam along $y$ direction. This is due to the fact that, at fixed value of $a$ increase in beam ellipticity (i.e., $\frac{a}{b}$ ) means the reduction in initial width of the beam along $y$ direction. Hence, with increase in beam ellipticity along $y$ direction makes the diffraction effect stronger along $y$ direction. This results in the reduced focusing of the laser beam along $y$ direction

It can also be seen that initially the increase in beam ellipticity does not produce any significant effect on self focusing of the beam along $x$ direction. However, as the beam penetrates deeper into the plasma the focusing along $x$ direction also decreases. This is due to the fact that as the beam penetrates deeper and deeper into the plasma, the nonlinear coupling between the two beam widths becomes stronger and stronger.

Fig.9 illustrates the effect of slope of density ramp on self focusing of the laser beam along the two transverse directions. It can be seen that with increase in the slope of density ramp the extent of self 
focusing of the laser beam along both the transverse directions increases. This is due to the fact that with increase in slope density of ramp, the number of electrons contributing to the ponderomotive nonlinearity increases along the direction of propagation. This results in enhanced transverse as well as longitudinal gradient in the index of refraction of plasma that in turn increases the extent of self focusing of the laser beam along the two transverse directions.

\section{Evolution of Axial Phase of Laser Beam}

During the propagation of a laser beam through a nonlinear medium its axial phase changes due to its transverse spatial confinement resulting as a consequence of its nonlinear refraction. The transverse spatial confinement through the position momentum uncertainty gives an additional transverse momentum to the photons of the beam and thus changes the longitudinal momentum the photons and hence the axial phase of the beam.

The overall wave number of the beam is related to its components through[39,40]

$$
k_{0}^{2}=k_{x}^{2}+k_{y}^{2}+k_{z}^{2}
$$

Defining the effective axial propagation constant for the laser beam weighted average as

$$
\overline{k_{z}}=k_{0}-\frac{<k_{x}^{2}>}{k_{0}}-\frac{<k_{y}^{2}>}{k_{0}}
$$

This effective propagation constant is associated with overall phase $\left(\theta_{p}\right)$ of the beam as

$$
\frac{d \theta_{p}}{d z}=k_{0}-\frac{<k_{x}^{2}>}{k_{0}}-\frac{<k_{y}^{2}>}{k_{0}}
$$

The first term in this equation gives the phase $k_{0} z$ of an infinite plane wave propagating along $z$ axis. However, the second term gives the axial phase shift

$$
\frac{d \theta}{d \xi}=-a^{2}\left(<k_{x}^{2}>+<k_{y}^{2}>\right)
$$

where,

$$
<k_{x, y}^{2}>=\frac{1}{I_{k}} \int_{-\infty}^{\infty} \int_{-\infty}^{\infty} k_{x, y}^{2} \tilde{A}_{0} \tilde{A}_{0}{ }^{\star} d k_{x} d k_{y}
$$




$$
\begin{gathered}
I_{k}=\int_{-\infty}^{\infty} \int_{-\infty}^{\infty} \tilde{A}_{0} \tilde{A}_{0}^{\star} d k_{x} d k_{y} \\
\tilde{A}_{0}\left(k_{x}, k_{y}\right)=\frac{1}{2 \pi} \int_{-\infty}^{\infty} \int_{-\infty}^{\infty} A_{0}(x, y, z) e r^{-\iota\left(k_{x} x+k_{y} y\right)} d x d y
\end{gathered}
$$

Eq.(23) gives the evolution of axial phase of the laser beam during its propagation through the plasma. We have solved this equation in association with eqs.(17) and (18), and the corresponding dynamics of the axial phase for different laser-plasma parameters has been depicted in figs.(10)-(12).

It is observed that axial phase $\theta$ decreases monotonically with distance of propagation, showing abrupt jumps at the periodic positions of the minimum beam widths. These jumps of axial phase at the focal positions of the laser beam give it a step like behaviour. The monotonic decrease in axial phase with distance is due to the fact that the self focusing of the laser beams with distance of propagation leads to reduction in volume of space available for its propagation. This in turn through position momentum uncertainty along the transverse directions

$$
\begin{aligned}
& \Delta x \Delta p_{x}=\text { Constant } \\
& \Delta y \Delta p_{y}=\text { Constant }
\end{aligned}
$$

results in increase in the transverse momentum of the photons of the laser beam. This situation is similar to that observed for a quantum particle trapped in a tube or a photon confined in a waveguide. However, the interesting fact is that in the present case there is no physical boundary to confine the photons. Now, as the overall momentum should remain conserved, the increase transverse momentum results in reduction in the longitudinal momentum of the photons. This reduction the longitudinal momentum is the consequence of monotonic decrease in the axial phase of the laser beam.

Step like behaviour of the axial phase, with each step occurring at positions of minimum beam width indicates that there is slowest decrement in $\theta$ at points of minimum beam width. This is opposite to the behaviour of phase in graded index fibers, where phase decreases slowest in the positions of minimum intensity i.e., maximum beam width. This difference in the behaviour of axial phase in 
plasmas and that in graded index fibers is due to the fact that due to their optical nonlinearity, plasmas behave as oscillating linear wave guides. In linear wave guides, the growth rate of axial phase is inversely proportional to the square of beam width.

Fig.10 depict that with increase in the value of deviation parameter $q$ there is decrease in the rate of change of axial phase with distance. This is due to the fact that as the spatial profile of the laser beam converges towards ideal Gaussian profile the transverse confinement of the laser beam due to self focusing decreases. As spatial confinement of the laser beam is homeostasis for the axial phase shift, the reduction of self focusing with increase in deviation parameter $q$ results in reduction in the rate of change of axial phase with distance. Thus, axial phase of the ideal Gaussian beams changes at slowest rate during their propagation through nonlinear media.

Fig.11 illustrates the effect of beam ellipticity on the evolution of axial phase of the laser beam. It can be seen that with increase in the ellipticity of the laser beam its axial phase changes at a slower rate. This is due to the reduction in overall focusing of the laser beam with increase in its ellipticity.

Fig.12 depicts the effect of slope of plasma density ramp on the axial phase of the laser beam. It can be seen that in denser plasmas the axial phase of the laser beam changes at a faster rate. This is due to the fact that denser is the plasma, higher is the spatial confinement of the laser beam due to self focusing. As a result the axial phase of the beam changes at a faster rate in denser plasmas.

\section{Conclusions}

In conclusion we have presented the effects of both ellipticity of the cross section of the laser beam as well as the deviation of amplitude structure from ideal Gaussian profile on its nonlinear propagation characteristics. It has been observed that although the deviation of amplitude structure from ideal Gaussian profile arises due to cavity imperfections, it helps to enhance the self focusing of the laser beam in nonlinear media. But along with enhancing the extent of self focusing it delays the focusing of the laser beam. It is also observed that initial ellipticity of the beam also plays a significant role 
in determining the propagation characteristics of the beam. Self focusing of the laser beam is lesser along the transverse direction along which it is initially more elliptical.

The results of present investigation may serve as a guide for the experimentalists working in the area of laser-plasma interactions and nonlinear optics.

\section{REFERENCES}

1. T. H. Maiman, "Stimulated optical radiation in Ruby," Nature, 187, 493 (1960).

2. E. Khalkhal, M. R. Tavirani, M. R. Zali and Z. Akbari, "The evaluation of laser application in surgery: a review article," J. Lasers Med. Sci. 10, 104 (2019).

3. C. Deutsch, H. Furukawa, K. Mima, M. Murakami and K. Nishihara, "Interaction physics of the fast ignitor concept," Phys. Rev. Lett. 77, 2483 (1996).

4. T. Tajima and J. M. Dawson, "Laser electron accelerator," Phys. Rev. 43, 267 (1979).

5. N. A. Roso, R. C. Moreira and J. B. Oliveira, "High power laser weapons and operational implications," J. Aerosp. Technol. Manag. 6, 231 (2014).

6. K. H. Kurniawan, M. o. Tjia and K. Kagawa, "Review of Laser-Induced Plasma, Its Mechanism, and Application to Quantitative Analysis of Hydrogen and Deuterium,” Appl. Spectroscopy Rev. 49, 323 (2014).

7. M. Leduc, J. Dugue and J. Simone, "Laser Cooling, Trapping, and Bose-Einstein Condensation of Atoms and Molecules," Phys. Today 71, 18 (2018).

8. R. Y. Chiao, E. Garmire and C. H. Townes, "Self-Trapping of Optical Beams", Phys. Rev. Lett. 13, $479(1965)$.

9. P. L. Kelley, "Self-focusing of optical beams," Phys. Rev. Lett. 15, 1005 (1966).

10. S. A. Akhmanov, A. P. Sukhorukov and R. V. Khokhlov, "Self-focusing and diffraction of light in a nonlinear medium," Usp. Fiz. Nauk. 93, 19 (1967).

11. G. A. Askaryan, "Effects of the Gradient of Strong Electromagnetic Beam on Electrons and Atoms," 
Soviet Phys. JETP 15, 1088 (1962).

12. M. D. Feit and J. A. Fleck, "Selftrapping of a laser beam in a cylindrical plasma column," Appl. Phys. Lett. 28, 121 (1976).

13. H. Hora, "Theory of relativistic self-focusing of laser radiation in plasmas," J. Opt. Soc. Am. 65, $882(1975)$

14. D. N. Gupta, M. S. Hur, I. Hwang, H. Suk and Ashok K. Sharma, "Plasma density ramp for relativistic self-focusing of an intense laser," J. Opt. Soc. Am. B 24, 1155 (2007).

15. A. Singh and K. Walia, "Self-focusing of Gaussian laser beam through collisionless plasmas and its effect on second harmonic generation," J. Fusion Ener. 30, 555 (2011).

16. A. Singh and N. Gupta, "Higher harmonic generation by self-focused q-Gaussian laser beam in preformed collisionless plasma channel," Laser and Part. Beams 32, 621 (2014).

17. Y. Wang, Y. Liang, J. Yao, C. Yuan and Z. Zhou, "Nonlinear propagation characteristics of multiGaussian beams in collisionless plasmas," J. Opt. Soc. Am. B 35, 3088 (2018).

18. El Sayed A. El Badawy, Mohamed S. Nagy, and Farag Z. El Halafawy, "Self-focusing of powerful CO2-laser beams in collisional plasmas," J. Opt. Soc. Am. 72, 1393 (1982).

19. D. N. Gupta, M. R. Islam, D. G. Jang, H. Suk and D. A. Jaroszynski, "Self-focusing of a high-intensity laser in a collisional plasma under weak relativistic-ponderomotive nonlinearity," Phys. Plasmas 20, $123103(2013)$.

20. M. Habibi and F. Ghamari, "Significant enhancement in self-focusing of high-power laser beam through dense plasmas by ramp density profile," J. Opt. Soc. Am. B 32, 1429 (2015).

21. N. Pathak, P. C. Agarwal, T. S. Gill and S. Kaur, "Characteristics of spatiotemporal dynamics of a quadruple Gaussian laser beam in a relativistic ponderomotive magnetized plasma," J. Opt. Soc. Am. B 37, $2892(2020)$.

22. G. Purohit, B. Gaur and P. Rawat, "Propagation of two intense cosh-Gaussian laser beams in plasma in the relativistic-ponderomotive regime," J. Opt. Soc. Am. B 33, 1716 (2016). 
23. S. D. Patil and M. V. Takale, "Self-focusing of Gaussian laser beam in weakly relativistic and ponderomotive regime using upward ramp of plasma density," Phys. Plasmas 20, 83101 (2013).

24. M. S. Sodha, A. K. Ghatak and V. K. Tripathi, "Progress in Optics.Volume XIII (edited by Wolf, E.)," North Holland, Amsterdam. 13, 169 (1976).

25. B. T. Spiers, M. P. Hill, C. Brown, L. Ceurvorst, N. Ratan, A. F. Savin, P. Allan, E. Floyd, J. Fyrth, L. Hobbs, S. James, J. Luis, M. Ramsay, N. Sircombe, J. Skidmore, R. Aboushelbaya, M. W. Mayr, R. Paddock, R. H. W. Wang and P. A. Norreys, ",Whole-beam self-focusing in fusion-relevant plasma," Phil. Trans. R. Soc. A. 379, 20200159 (2021).

26. N. Gupta and S. Kumar, "Linear and nonlinear propagation characteristics of multi-Gaussian laser beams," Chinese Phys. B 29, 114210 (2020).

27. T. Singh and S. S. Kaul, "Self-focusing and self-phase modulation of elliptic Gaussian laser beam in a graded Kerr-medium,” Indian J. Pure Appl. Phys. 37, 794797 (1999).

28. S. Konar and A. Sengupta, "Propagation of an elliptic Gaussian laser beam in a medium with saturable nonlinearity," J. Opt. Soc. Am. B 11, 1644 (1994).

29. F. Cornolti, M. Lucchesi and B. Zambon, "Elliptic Gaussian beam selffocusing in nonlinear media," Opt. Commun. 75, 129 (1990).

30. H. Kumar, M. Aggarwal, Richa and T. S. Gill, "Self-focusing of an elliptic-Gaussian laser beam in relativistic ponderomotive plasma using a ramp density profile," J. Opt. Soc. Am. B 35, 1635 (2018).

31. P. K. Patel, M. H. Key, A. J. Mackinnon, R. Berry, M. Borghesi, D. M. Chambers, H. Chen, R. Clarke, C. Damian, R. Eagleton, R. Freeman, S. Glenzer, G. Gregori, R. Heathcote, D. Hey, N. Izumi, S. Kar, J. King, A. Nikroo, A. Niles, H. S. Park, J. Pasley, N. Patel, R. Shepherd, R. A. Snavely, D. Steinman, C. Stoeckl, M. Storm, W. Theobald, R. Town, R. Van Maren, S. C. Wilks, and B. Zhang, "Integrated laser-target interaction experiments on the RAL petawatt laser," Plasma Phys. Controlled Fusion 47, B833 (2005).

32. M. Nakatsutsumi, J. R. Davies, R. Kodama, J. S. Green, K. L. Lancaster, K. U. Akli, F. N. Beg, S. N. 
Chen, D. Clark, R. R. Freeman, C. D. Gregory, H. Habara, R. Heathcote, D. S. Hey, K. Highbarger, P. Jaanimagi, M. H. Key, K. Krushelnick, T. Ma, A. MacPhee, A. J. MacKinnon, H. Nakamura, R. B. Stephens, M. Storm, M. Tampo, W. Theobald, L. Van Woerkom, R. L. Weber, M. S. Wei, N. C. Woolsey, and P. A. Norreys, "Space and time resolved measurements of the heating of solids to ten million kelvin by a petawatt laser," New J. Phys. 10, 043046 (2008).

33. C. Tsallis, "Nonadditive entropy and nonextensive statistical mechanics-an overview after 20 years," Braz. J. Phys. 39, 337 (2009).

34. M. Yadav, D. N. Gupta and S. C. Sharma, "Electron plasma wave excitation by a q-Gaussian laser beam and subsequent electron acceleration," Phys.Plasmas 27, 093106 (2020).

35. A. Sharma and I. Kourakis, "Spatial evolution of a q-Gaussian laser beam in relativistic plasma," Laser and Part. Beams 28, 479 (2010).

36. N. Gupta and S. Kumar, "Generation of second harmonics of q -Gaussian laser beams in collisional plasma with upward density ramp," Laser Phys. 30, 066003 (2020).

37. D. Anderson, M. Bonnedal and M. Lisak, "Nonlinear propagation of elliptically shaped Gaussian laser beams," 23, 115 (1980).

38. D. Anderson and M. Bonnedal, Variational approach to nonlinear self-focusing of Gaussian laser beams, Phys. Fluids 22, 105 (1979).

39. S. Feng and H. G. Winful, "Physical origin of the Gouy phase shift," Opt. Lett. 26, 485 (2001).

40. P. Hariharan and P. A. Robinson, "The gouy phase shift as a geometrical quantum effect," J. Mod. Opt. 43, 219. (1996). 


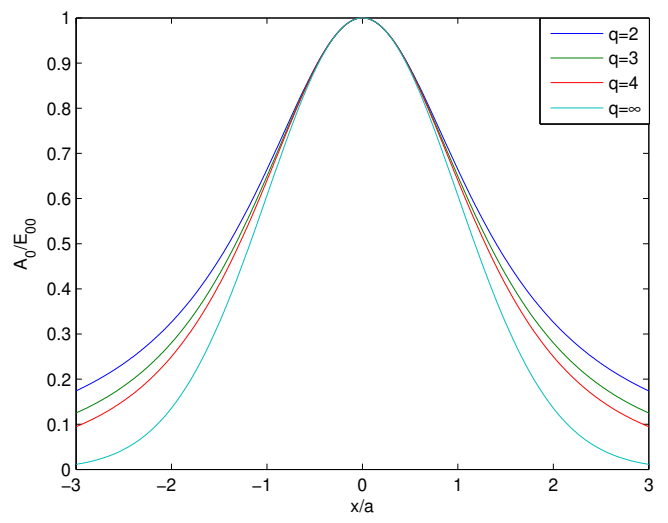

Figure 1: Variation of axial amplitude with transverse coordinate $\frac{x}{a}$ for different values of deviation parameter $q$. 
(a)

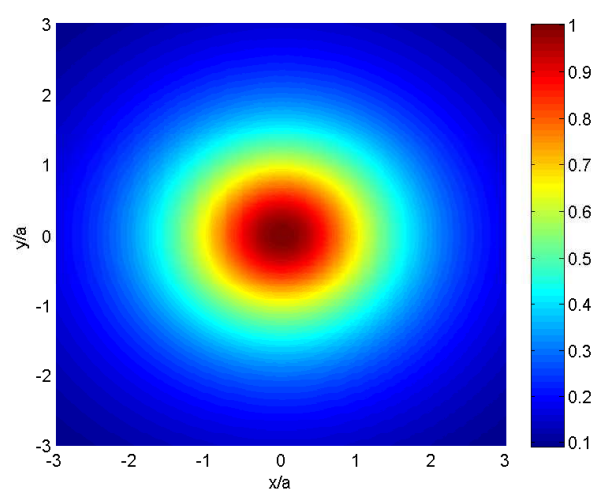

(c)

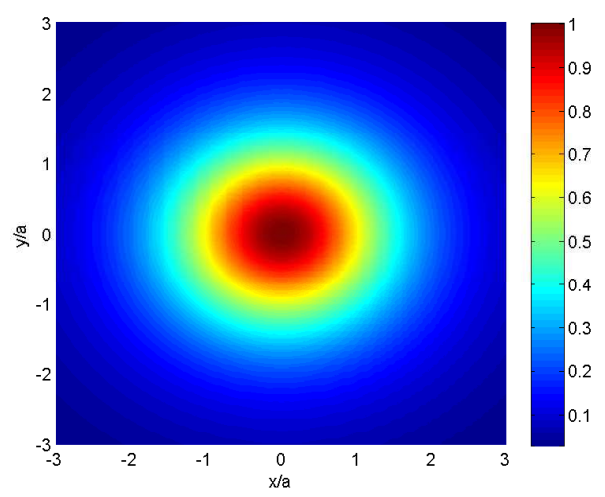

(b)

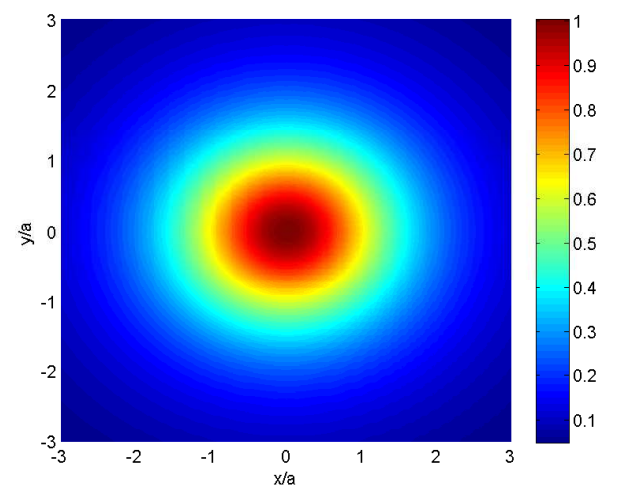

(d)

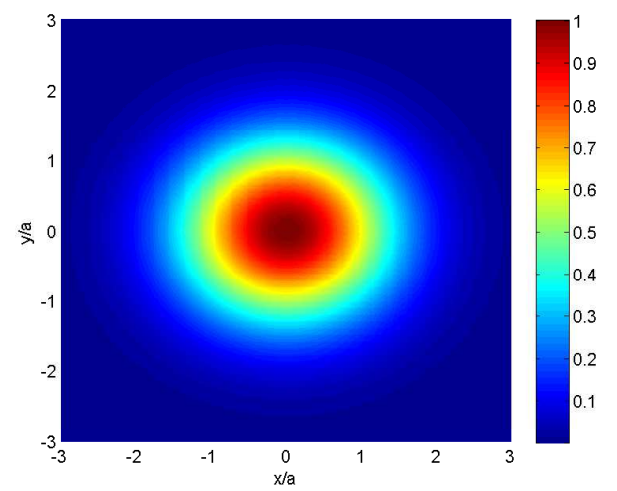

Figure 2: Contour plots for amplitude structure of elliptical $q$-Gaussian laser beams for $\frac{a}{b}=1.1$ and for different values of deviation parameter $q$ viz., (a) $q=2$, (b) $q=3$, (c) $q=4$ and (d) $q=\infty$ 


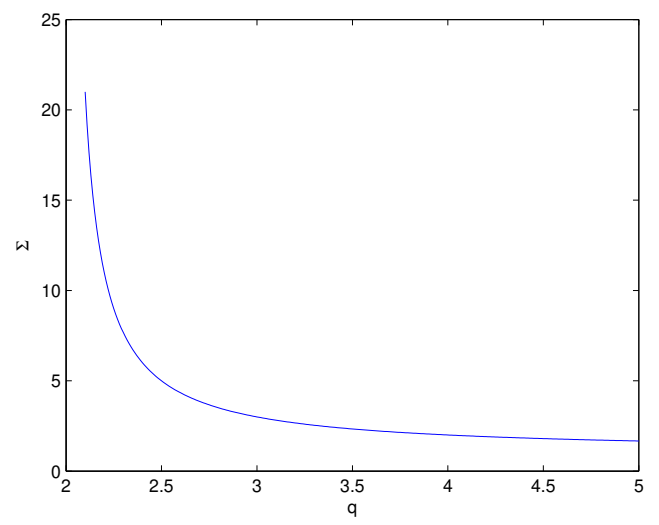

Figure 3: Variation of effective beam width of $q$-Gaussian laser beam with deviation parameter $q$.

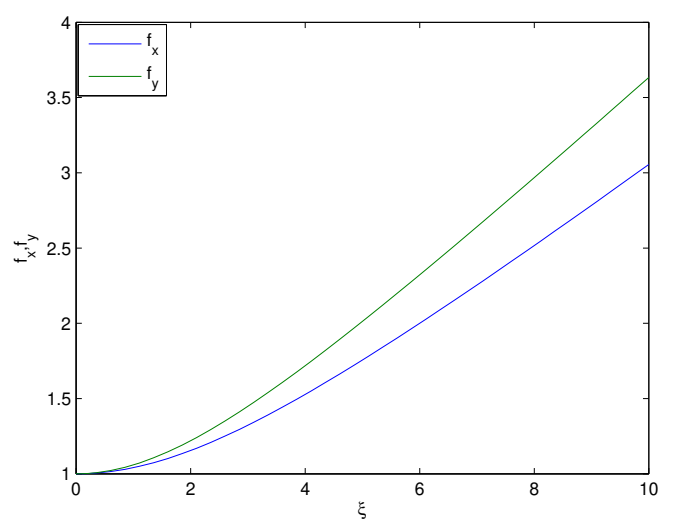

Figure 4: Variation of beam width parameters $f_{x, y}$ with distance of propagation in vacuum for fixed ellipticity $\frac{a}{b}=1.1$. 

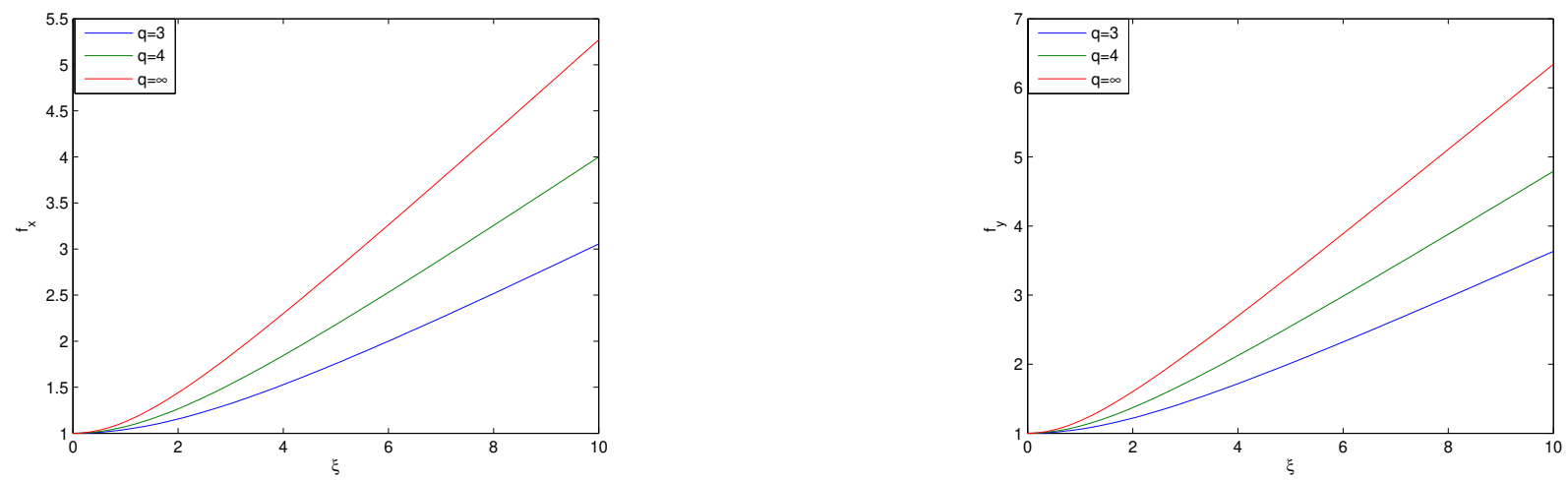

Figure 5: Variation of beam width parameters $f_{x, y}$ with distance of propagation in vacuum for different values deviation parameter $q$ and at fixed ellipticity $\frac{a}{b}=1.1$.

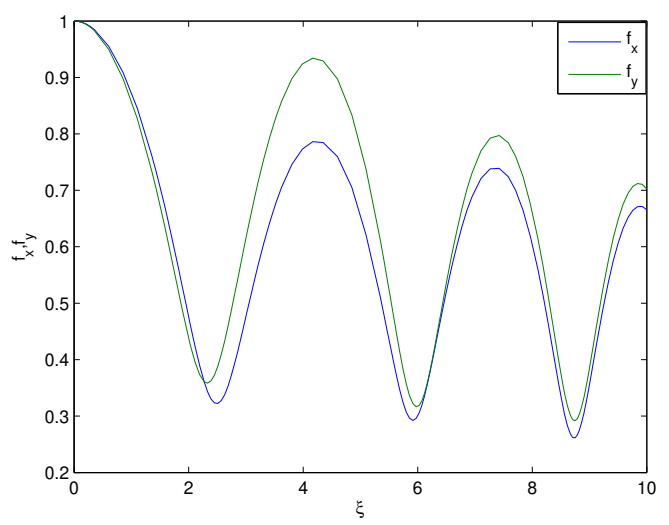

Figure 6: Variation of beam width parameters $f_{x, y}$ with distance of propagation in plasma for $q=3, d^{\prime}=0.25$ and $\frac{a}{b}=1.1$. 

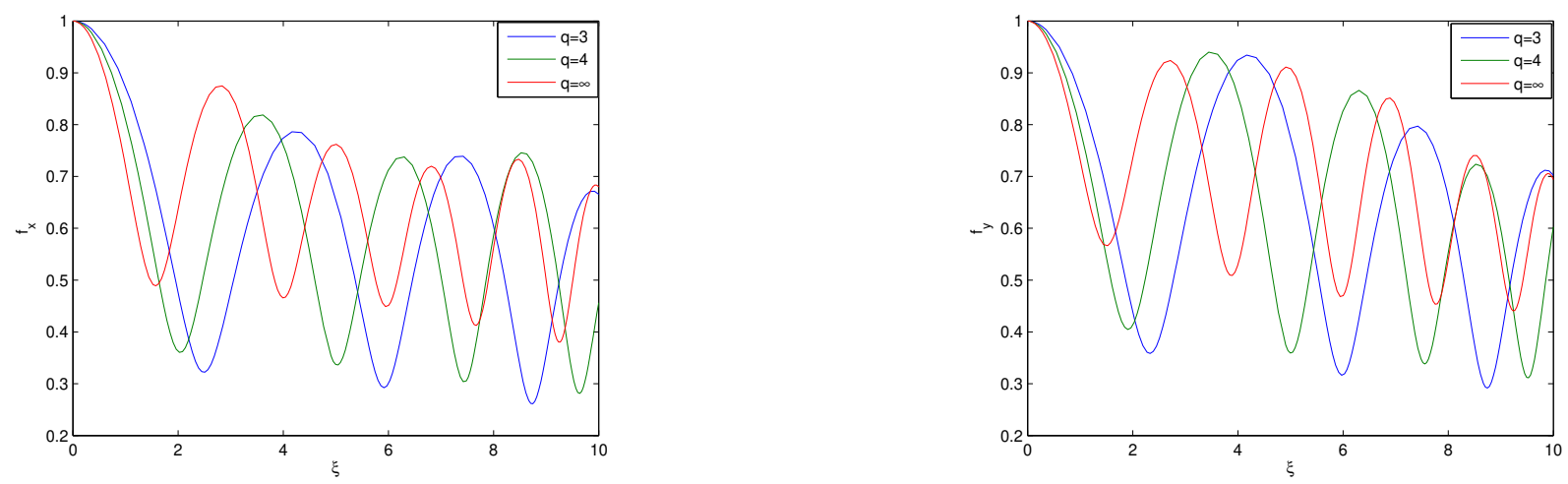

Figure 7: Variation of beam width parameters $f_{x, y}$ with distance of propagation in plasma for $q=(3,4, \infty), d^{\prime}=0.25$ and $\frac{a}{b}=1.1$.
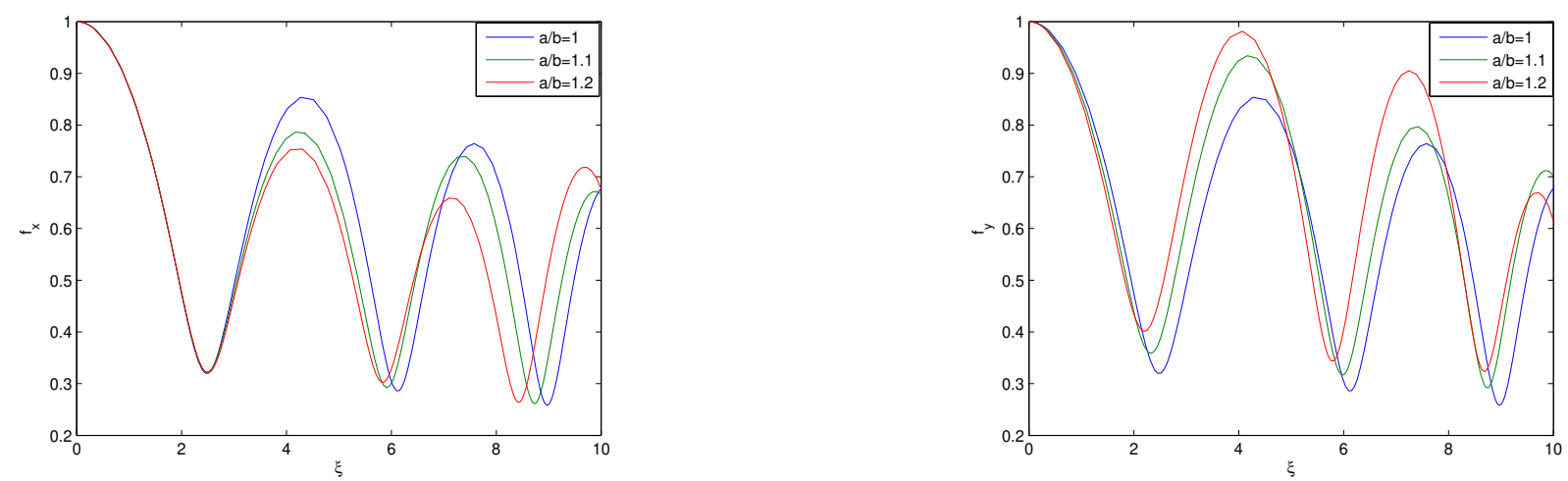

Figure 8: Variation of beam width parameters $f_{x, y}$ with distance of propagation in plasma for $q=3, d^{\prime}=0.25$ and $\frac{a}{b}=(1,1.1,1.2)$. 

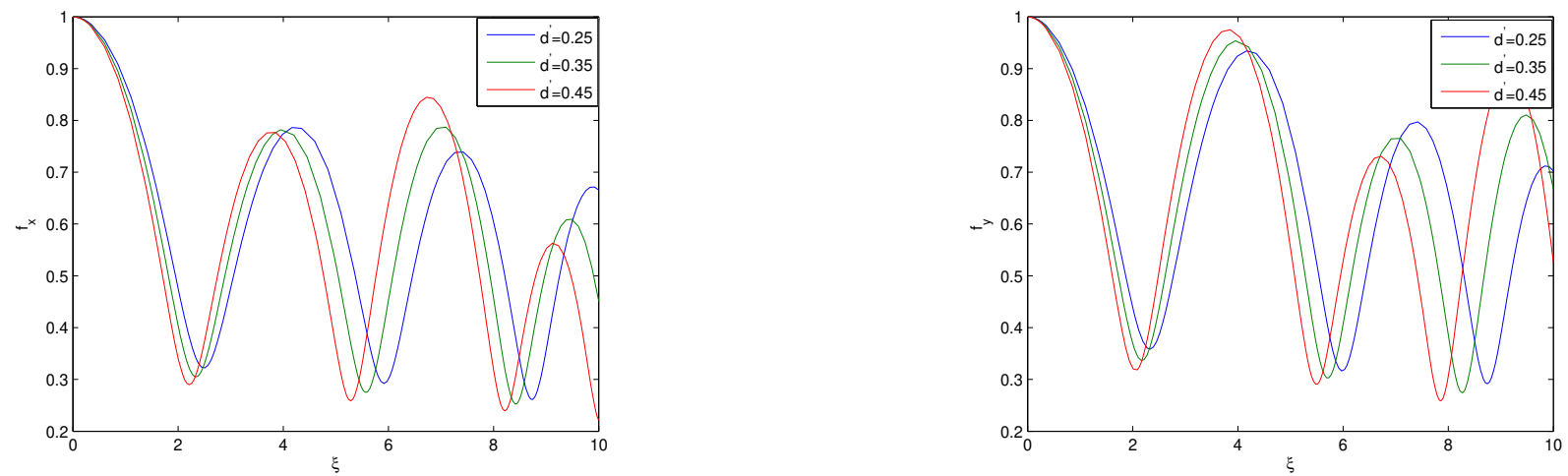

Figure 9: Variation of beam width parameters $f_{x, y}$ with distance of propagation in plasma for $q=3, d^{\prime}=(0.25,0.35,0.45)$ and $\frac{a}{b}=1.1$.

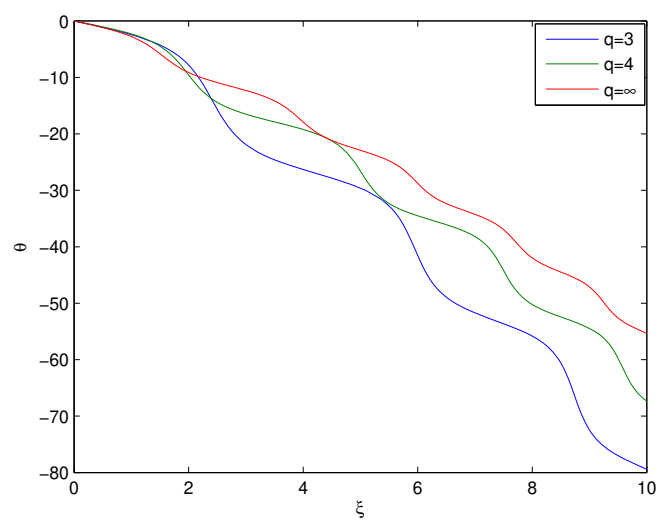

Figure 10: Variation of axial phsae $\theta$ with distance of propagation in plasma for $q=(3,4, \infty)$, $d^{\prime}=0.25$ and $\frac{a}{b}=1.1$. 


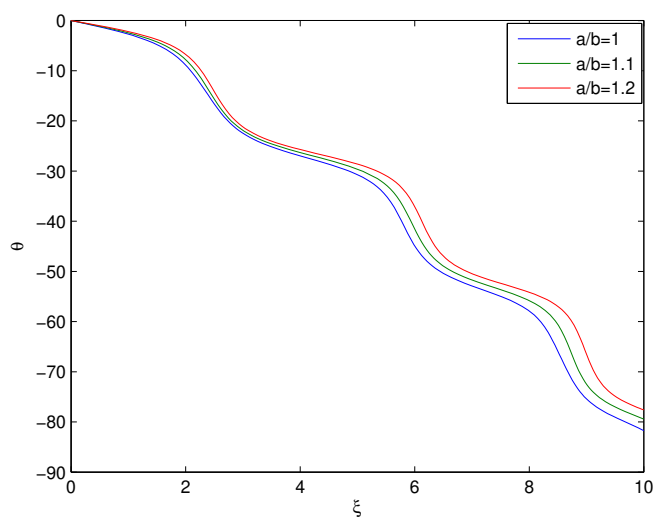

Figure 11: Variation of axial phsae $\theta$ with distance of propagation in plasma for $q=3, d^{\prime}=0.25$ and $\frac{a}{b}=(1,1.1,1.2)$.

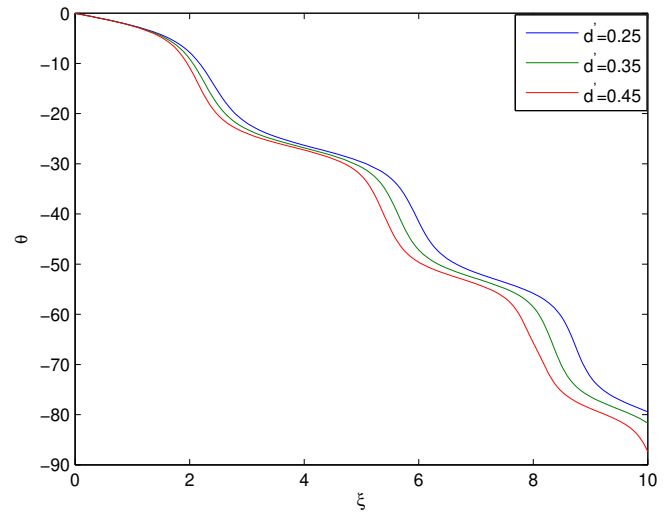

Figure 12: Variation of axial phsae $\theta$ with distance of propagation in plasma for $q=3, d^{\prime}=$ $(0.25,0.35,0.45)$ and $\frac{a}{b}=1.1$ 
Figures

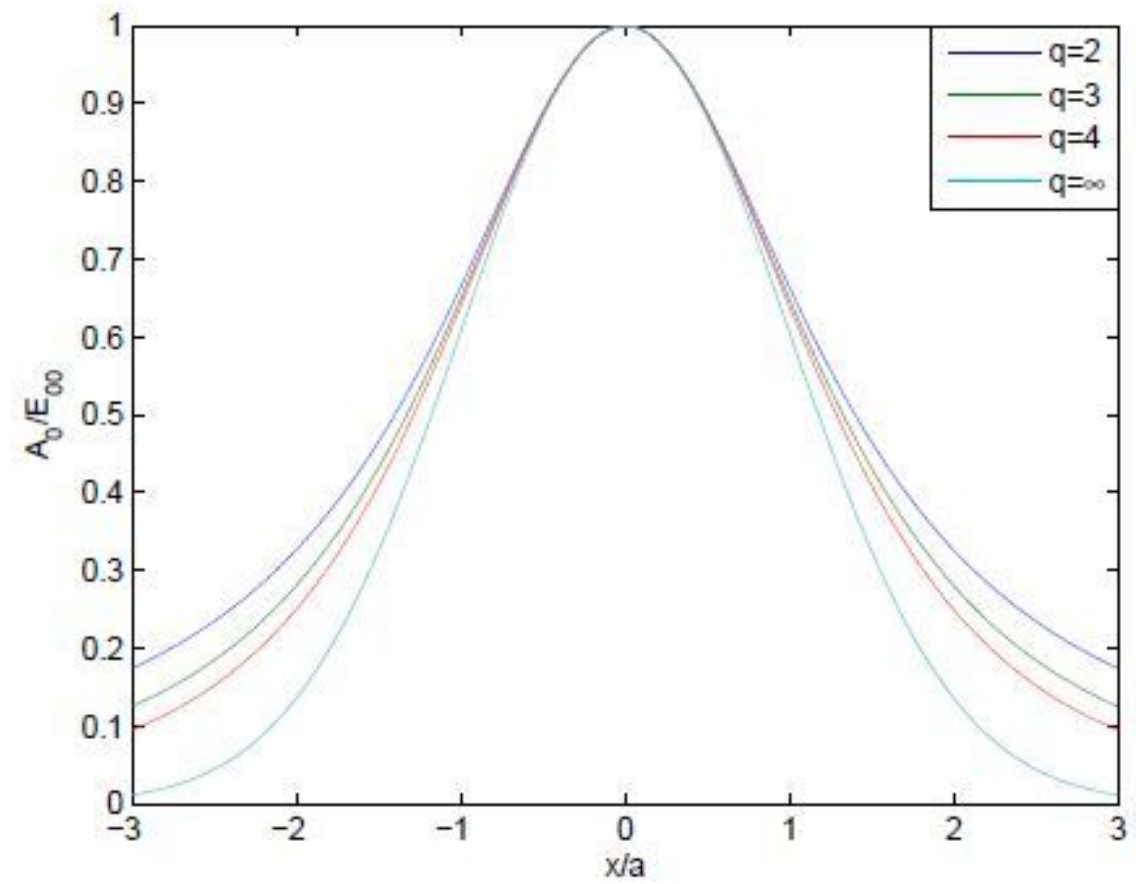

\section{Figure 1}

Variation of axial amplitude with transverse coordinate $\mathrm{x} / \mathrm{a}$ for different values of deviation parameter $\mathrm{q}$. 
(a)

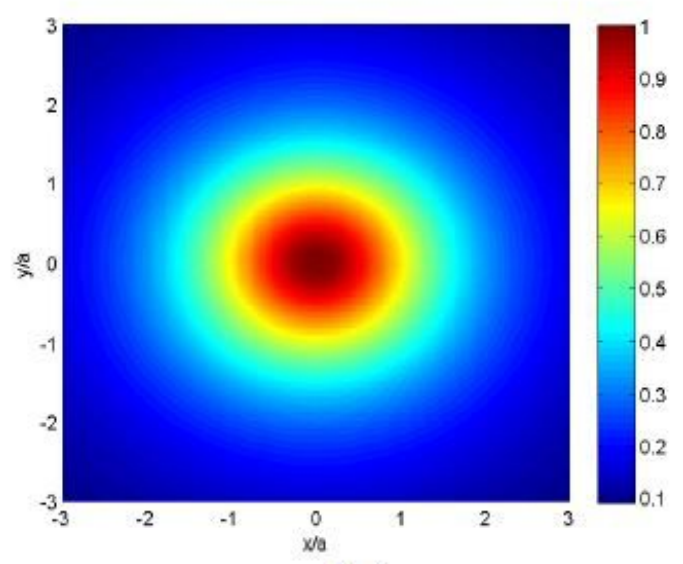

(c)

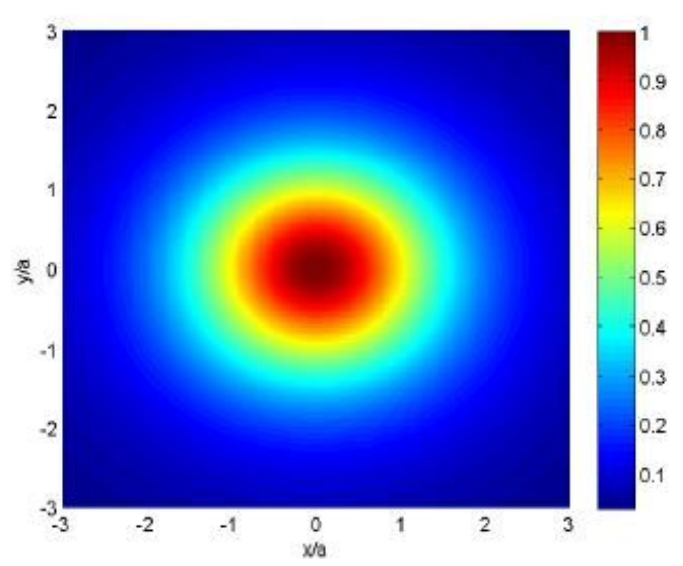

(b)

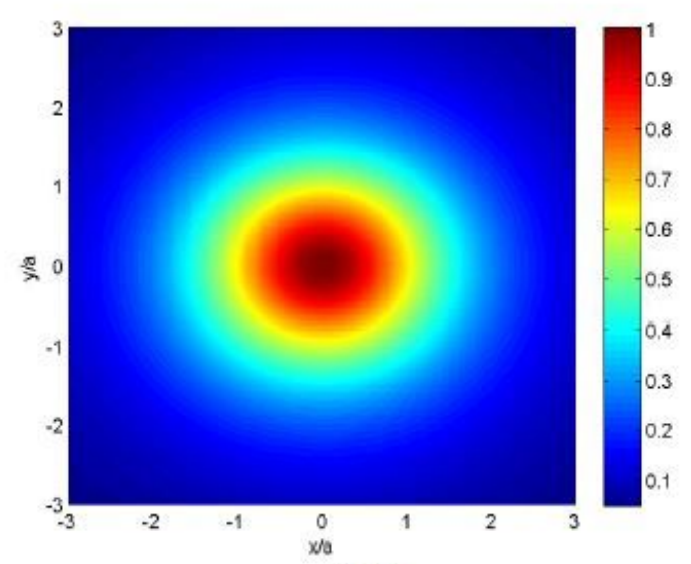

(d)

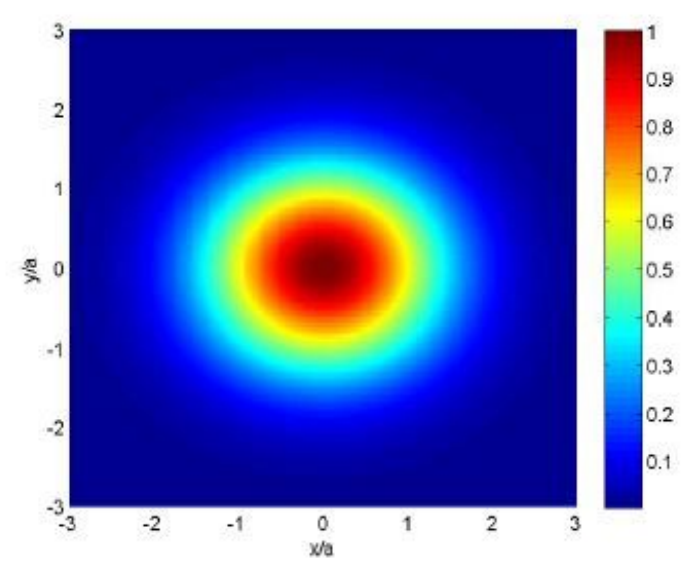

Figure 2

Due to technical limitations, the caption for this figure is only available in the manuscript. 


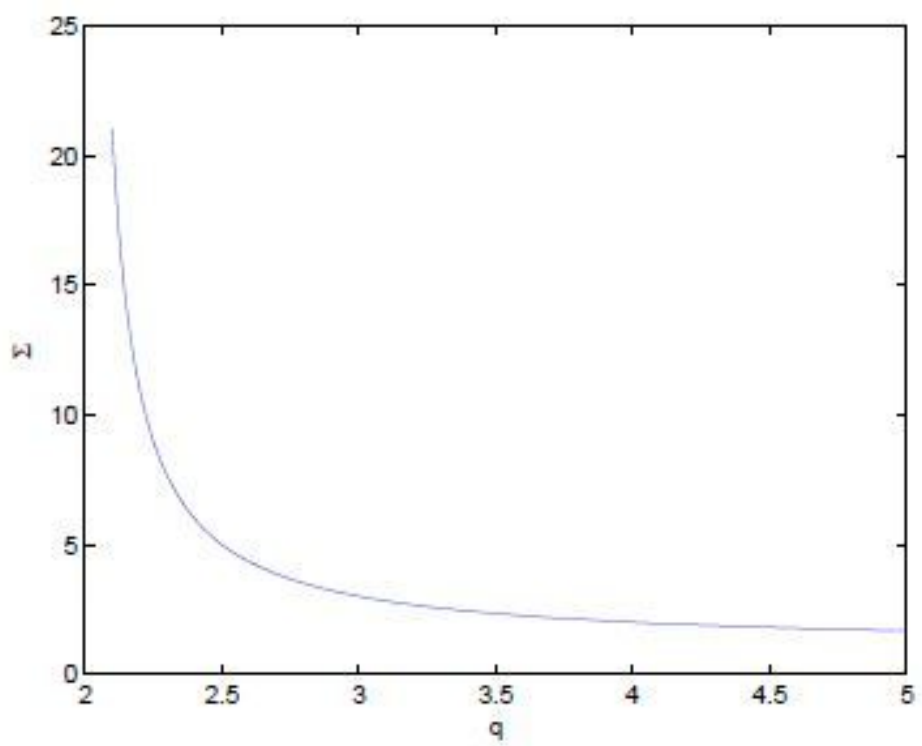

Figure 3

Variation of effective beam width of q-Gaussian laser beam with deviation parameter q.

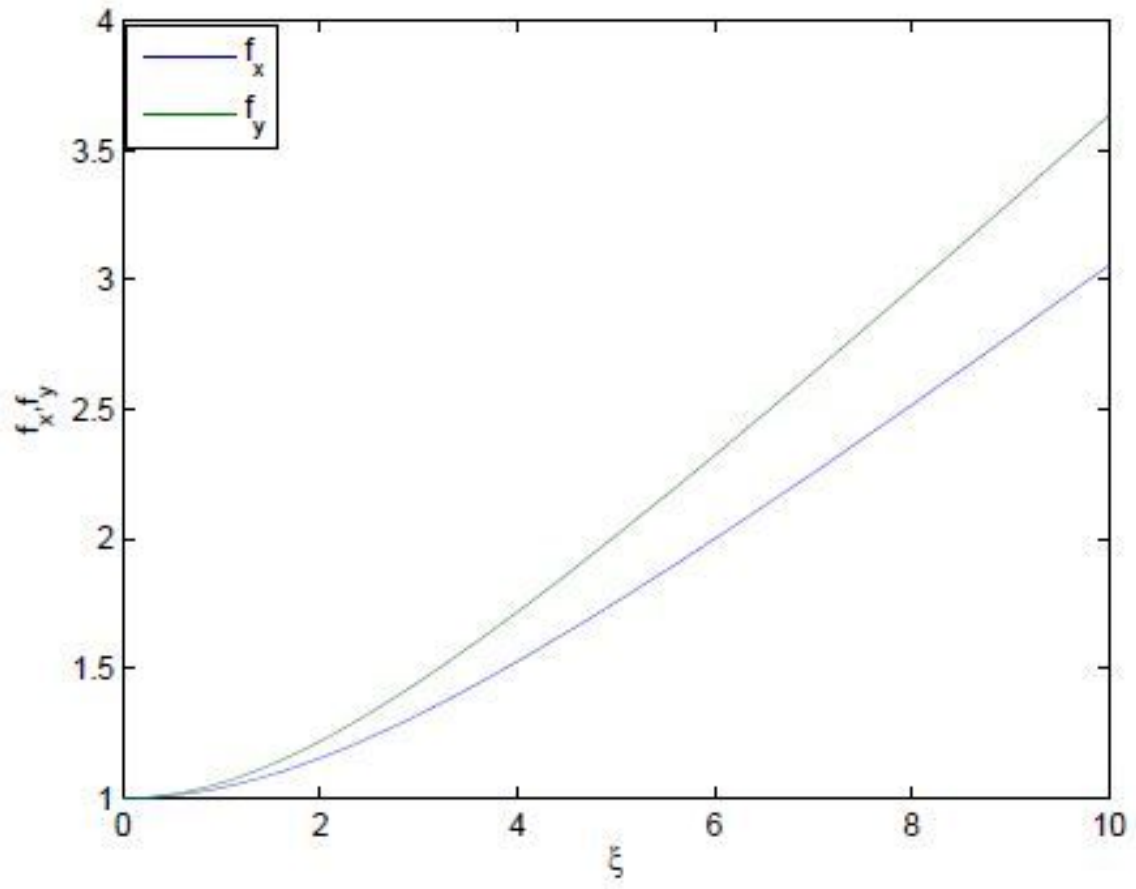

\section{Figure 4}

Variation of effective beam width of q-Gaussian laser beam with deviation parameter q. 

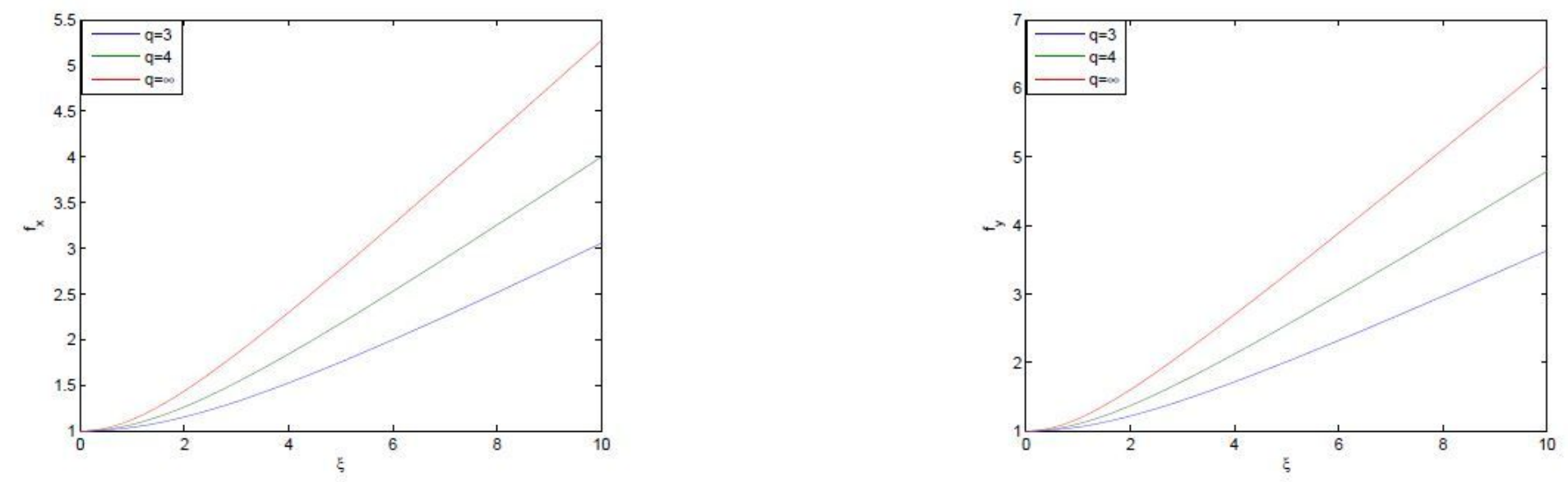

Figure 5

Due to technical limitations, the caption for this figure is only available in the manuscript.

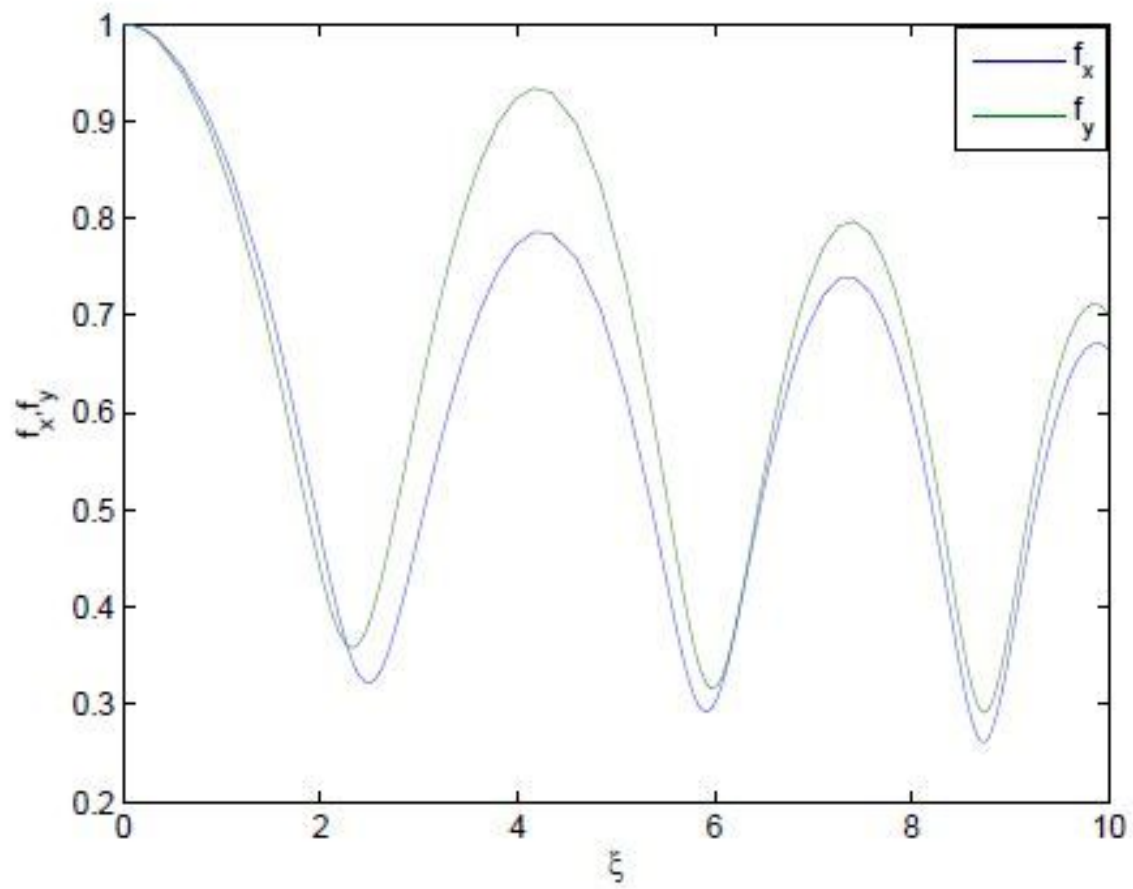

Figure 6

Due to technical limitations, the caption for this figure is only available in the manuscript. 

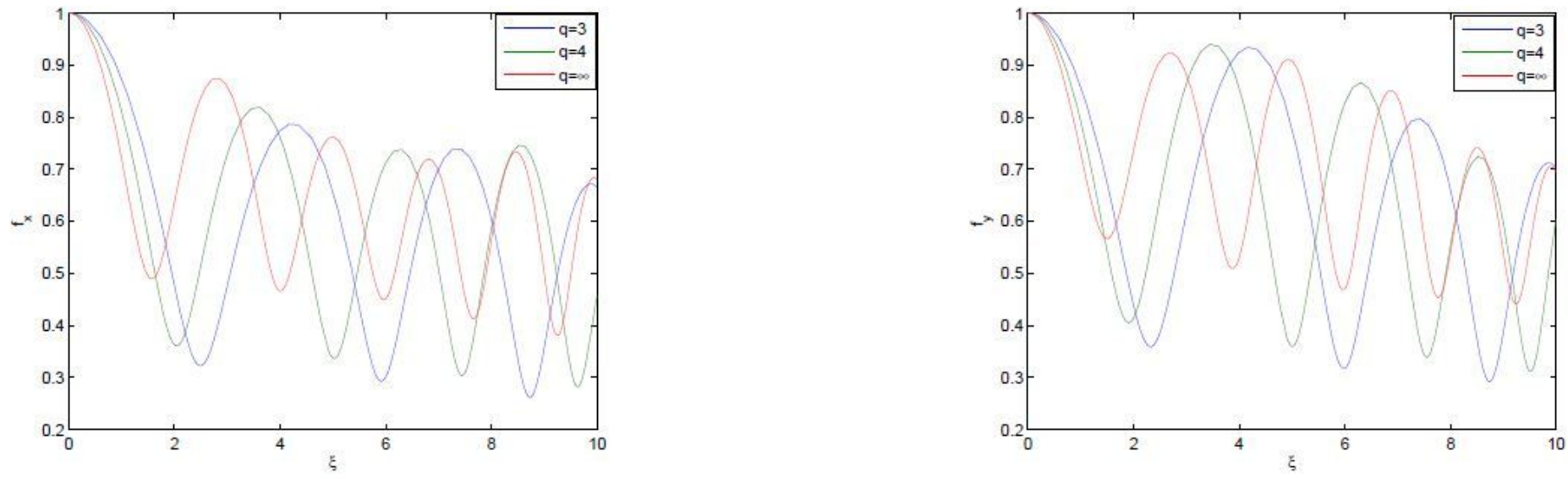

Figure 7

Due to technical limitations, the caption for this figure is only available in the manuscript.
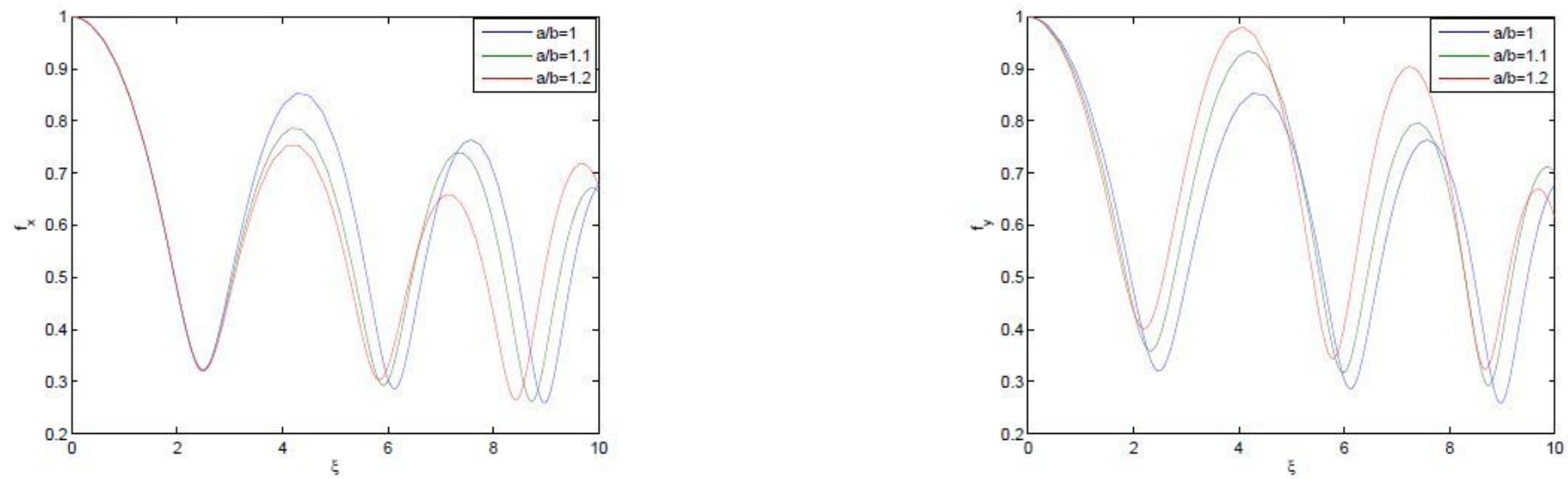

\section{Figure 8}

Due to technical limitations, the caption for this figure is only available in the manuscript.
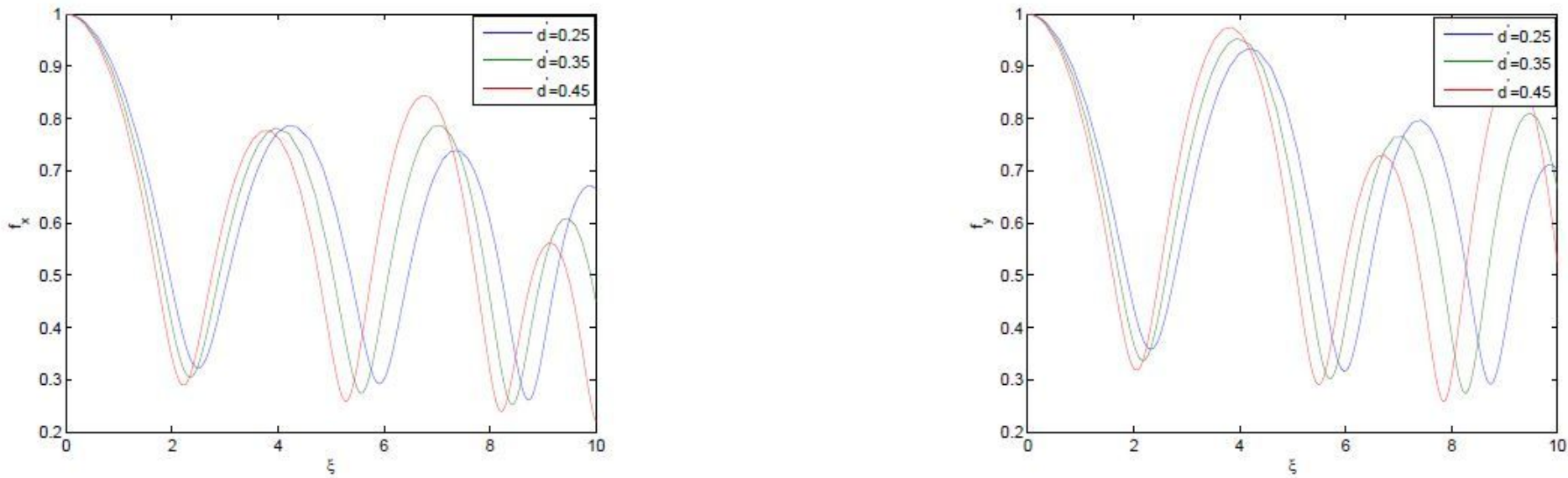

Figure 9 
Due to technical limitations, the caption for this figure is only available in the manuscript.

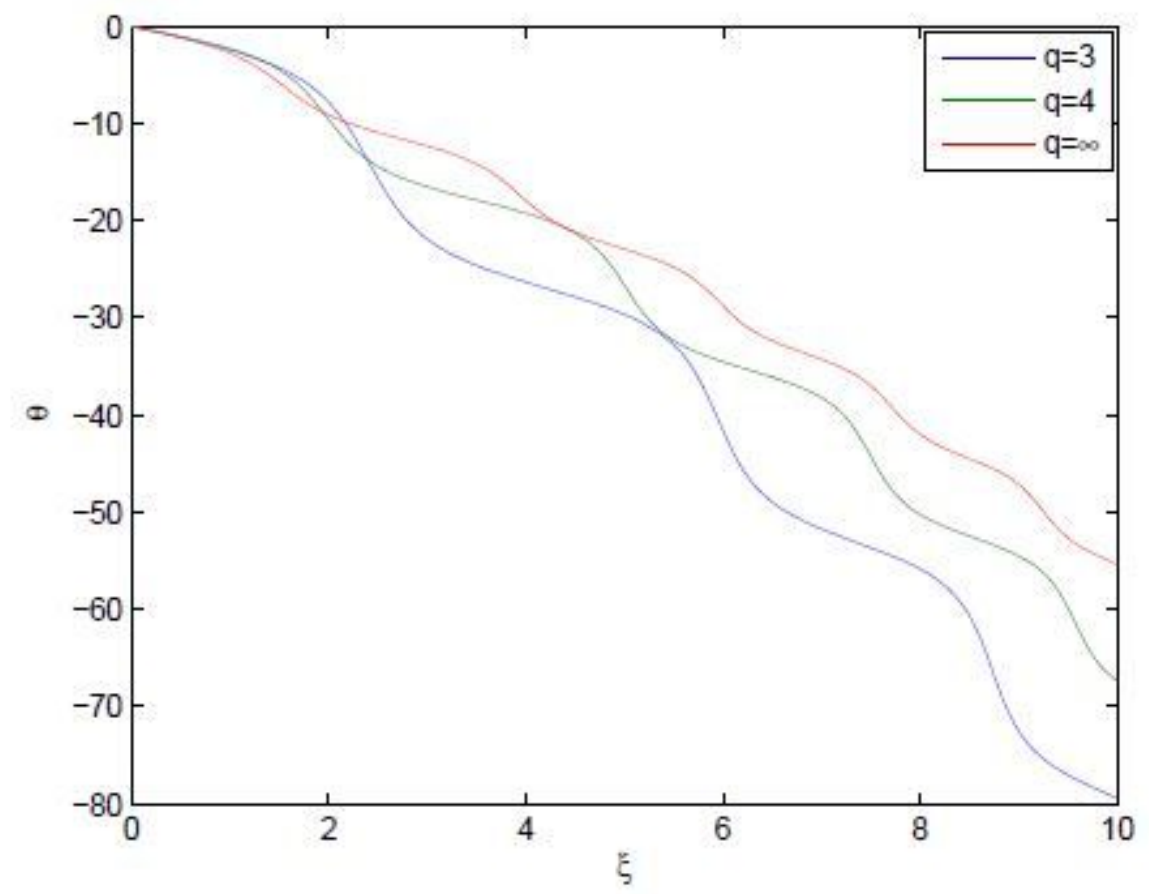

Figure 10

Due to technical limitations, the caption for this figure is only available in the manuscript.

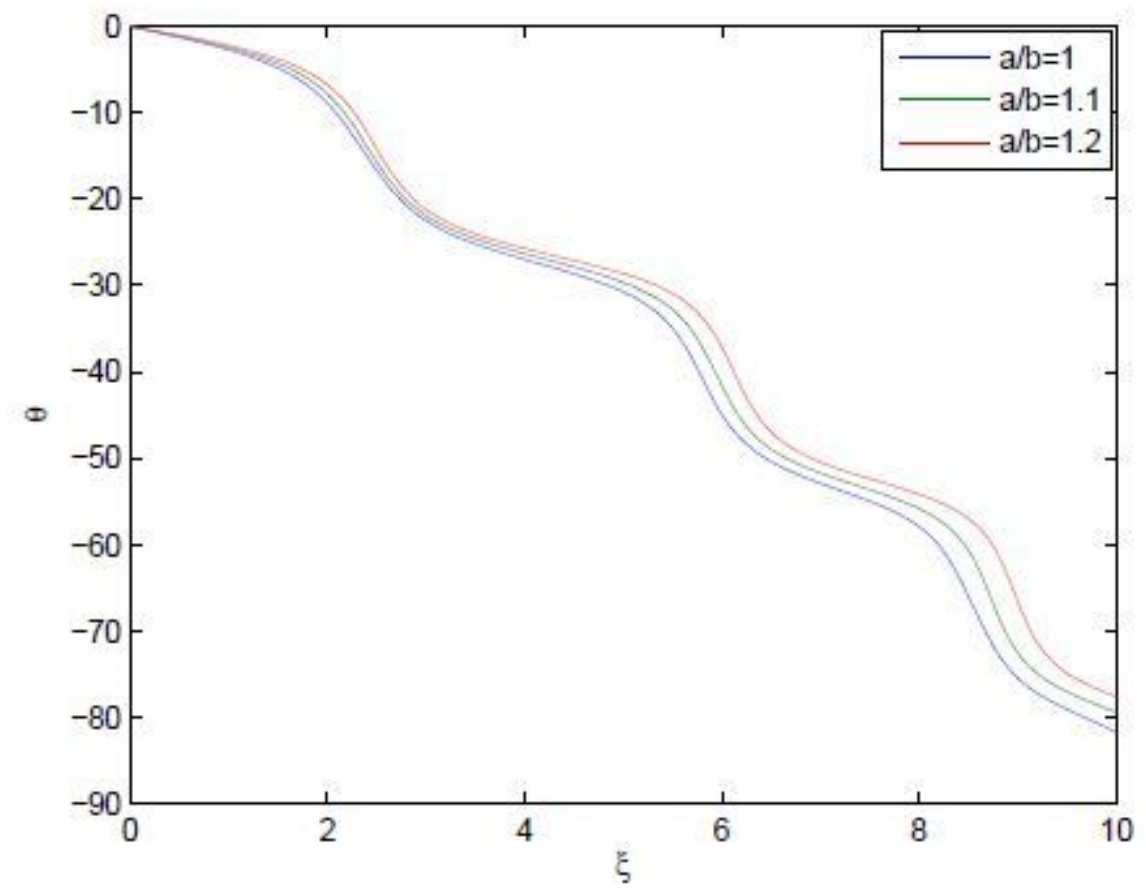

Figure 11 
Due to technical limitations, the caption for this figure is only available in the manuscript.

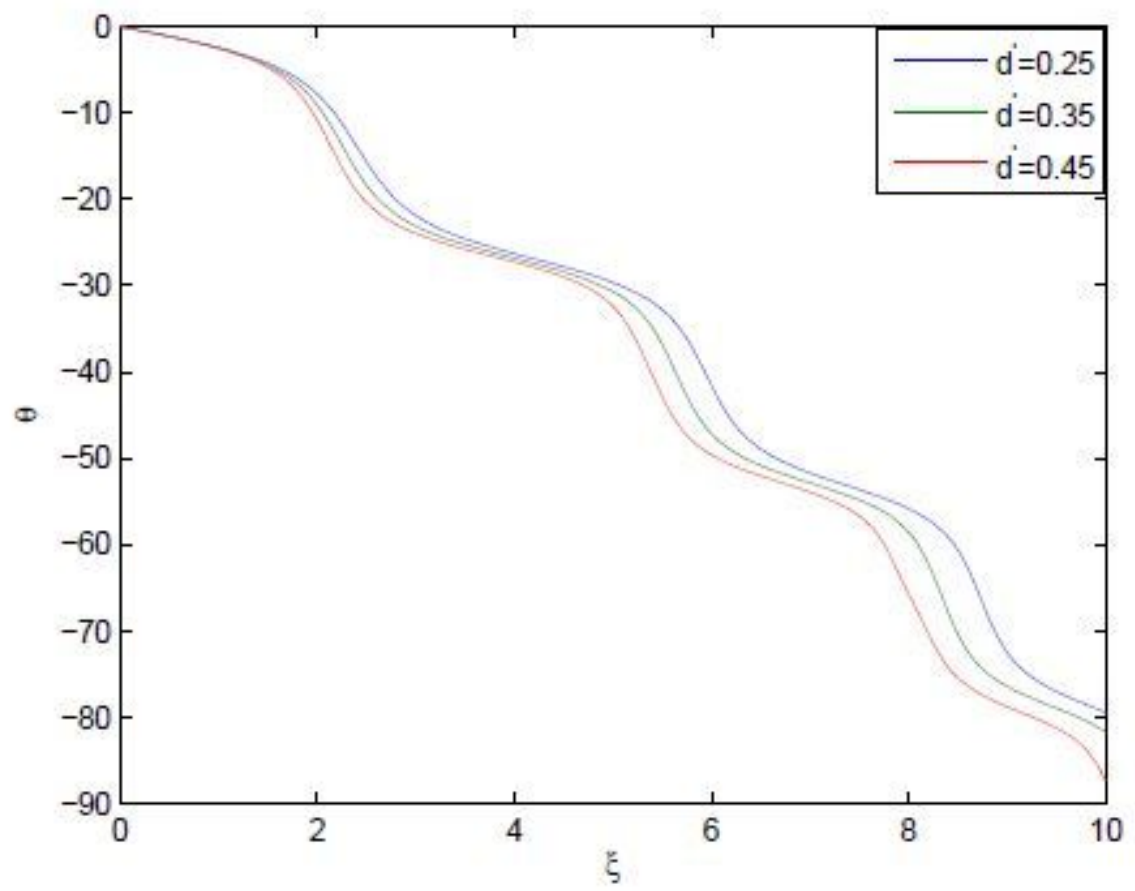

Figure 12

Due to technical limitations, the caption for this figure is only available in the manuscript. 